\title{
Energy Efficiency Optimization for Spatial Switching-based MIMO SWIPT System
}

DOI:

10.1109/VTCSpring.2017.8108293

\section{Document Version}

Accepted author manuscript

Link to publication record in Manchester Research Explorer

\section{Citation for published version (APA):}

Tang, J., So, K. C., Shojaeifard, A., \& Wong, K-K. (2017). Energy Efficiency Optimization for Spatial Switchingbased MIMO SWIPT System. In IEEE Vehicular Technology Conference Spring 2017 (7 ed., Vol. 16, pp. 47544769). IEEE. https://doi.org/10.1109/VTCSpring.2017.8108293

\section{Published in:}

IEEE Vehicular Technology Conference Spring 2017

\section{Citing this paper}

Please note that where the full-text provided on Manchester Research Explorer is the Author Accepted Manuscript or Proof version this may differ from the final Published version. If citing, it is advised that you check and use the publisher's definitive version.

\section{General rights}

Copyright and moral rights for the publications made accessible in the Research Explorer are retained by the authors and/or other copyright owners and it is a condition of accessing publications that users recognise and abide by the legal requirements associated with these rights.

\section{Takedown policy}

If you believe that this document breaches copyright please refer to the University of Manchester's Takedown Procedures [http://man.ac.uk/04Y6Bo] or contact uml.scholarlycommunications@manchester.ac.uk providing relevant details, so we can investigate your claim.

\section{OPEN ACCESS}




\title{
Energy Efficiency Optimization for Spatial Switching-based MIMO SWIPT System
}

\author{
Jie Tang ${ }^{1}$, Daniel K. C. So ${ }^{2}$, Arman Shojaeifard ${ }^{3}$ and Kai-Kit Wong ${ }^{3}$ \\ ${ }^{1}$ South China University of Technology, Email: eejtang@scut.edu.cn \\ ${ }^{2}$ University of Manchester, Email: d.so@manchester.ac.uk \\ ${ }^{3}$ University College London, Email: \{a.shojaeifard, kai-kit.wong\}@ucl.ac.uk.
}

\begin{abstract}
In this paper, we investigate joint antenna selection and spatial switching (SS) for energy efficiency (EE) optimization in a multiple-input multiple-output (MIMO) simultaneous wireless information and power transfer (SWIPT) system. A practical linear power model taking into account the entire transmit-receive chain is accordingly utilized. The corresponding fractional-combinatorial and non-convex EE problem, involving joint optimization of eigen-channel assignment, power allocation, and active receive antenna set selection, subject to satisfying minimum sum-rate and power transfer constraints, is extremely difficult to solve directly. In order to tackle this, we separate the eigen-channel assignment and power allocation procedure with the antenna selection functionality. In particular, we first tackle the EE maximization problem under fixed receive antenna set using Dinkelbach-based convex programming. We then provide a fundamental study of the achievable $\mathrm{EE}$ with antenna selection and accordingly develop dynamic optimal exhaustive search and Frobenius-norm-based schemes. Simulation results confirm the theoretical findings and demonstrate that the proposed resource allocation algorithms can efficiently approach the optimal EE.
\end{abstract}

\section{INTRODUCTION}

Ambient radio frequency (RF) signals can be used in conjunction with transmitting information to transfer power with adequate efficiency over relatively short transmit-receive distances. As a result, wireless power transfer (WPT) and energy harvesting $(\mathrm{EH})$ have recently emerged as promising candidate solutions for jointly improving energy efficiency (EE) and prolonging battery-life in fifth-generation (5G) and beyond communication systems. Simultaneous wireless information and power transfer (SWIPT) is considered particularly attractive for small-cell networks [1].

The information theoretic bounds for a single-input singleoutput (SISO) SWIPT system was investigated in [2]. Motivated by this, the authors in [3] investigated practical beamforming techniques in a multiple-input multiple-output (MIMO) SWIPT system and proposed two potential receiver design strategies, namely time-switching (TS), power-splitting (PS). Based on these, many recent research works have been carried out considering different system aspects. The focus in most existing works on SWIPT systems has been placed on maximizing either the throughput or the harvested energy [4], [5]. However, designing systems with the sole goal of spectral efficiency (SE) maximization constitutes to ever-rising network power consumption, which goes against global commitments for sustainable development. Meanwhile, the alternative approach to gain as much harvested energy as possible adversely affects information transfer, leading to the degradation of system quality of service (QoS).

In this paper, we provide a fundamental study of the EE optimization problem considering spatial-switching (SS)-based MIMO SWIPT system. Our aim is to maximize the EE under minimum sum-rate and power transfer constraints, by jointly optimizing receive active antenna set selection, eigenchannel assignment, and power allocation. The EE optimization problem under consideration is extremely difficult to tackle directly, given that it is fractional-combinatorial and non-convex. In order to tackle this problem, we propose a duallayer approach where the antenna selection procedure is separated with the eigen-channel allocation and power allocation operation. We provide simulation results in order to confirm the validity of our theoretical findings and draw design insights into the performance of SS-baed MIMO SWIPT systems.

\section{PReliminaries}

We consider a point-to-point MIMO SWIPT system where the source and the destination are respectively equipped with $N_{T}$ transmit antennas and $N_{R}$ receive antennas, as shown in Fig. 1. We assume a constant power supply is connected to the source, whilst the destination is capable of harvesting and transferring RF energy. In the context of SS-based receiver, the MIMO channel can be decomposed using SVD with the corresponding eigen-channels being used either to convey information or to transfer energy [5].

Intuitively, employing more receive antennas allows for achieving higher sum-rate and harvested energy. This however comes at the cost of larger transmit-independent power consumption. As a result of this trade-off, fully utilizing all available receive antennas with SS receiver does not necessarily correspond to an energy efficient strategy. In fact, the appropriate selection of the active receive antenna set through activation/deactivation of the corresponding RF chain switches is essential towards achieving high EE. With all receive antennas active, the channel matrix from the source to destination is denoted with $\mathbf{H} \in C^{N_{R} \times N_{T}}$. In this work, we consider an uncorrelated flat-fading MIMO Rayleigh channel model. As a result, with the number of active receive antennas $N$, 


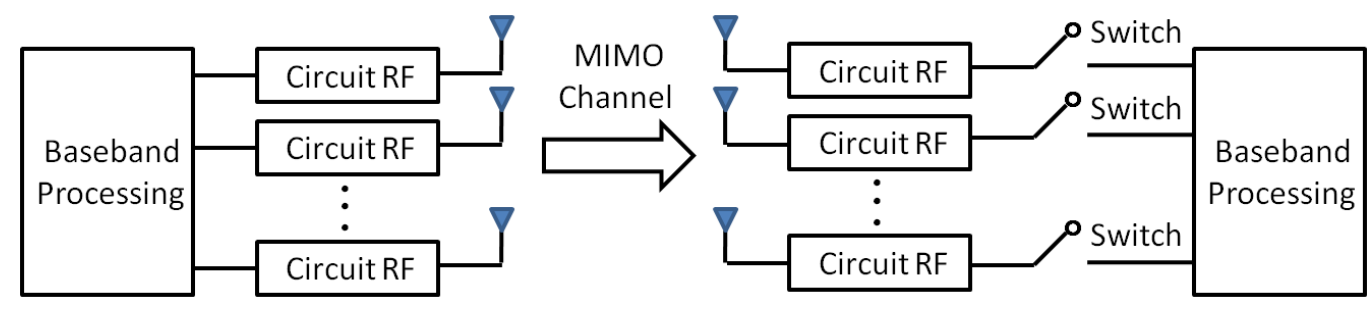

Fig. 1. Schematic example of a point-to-point MIMO system with SS-based receiver.

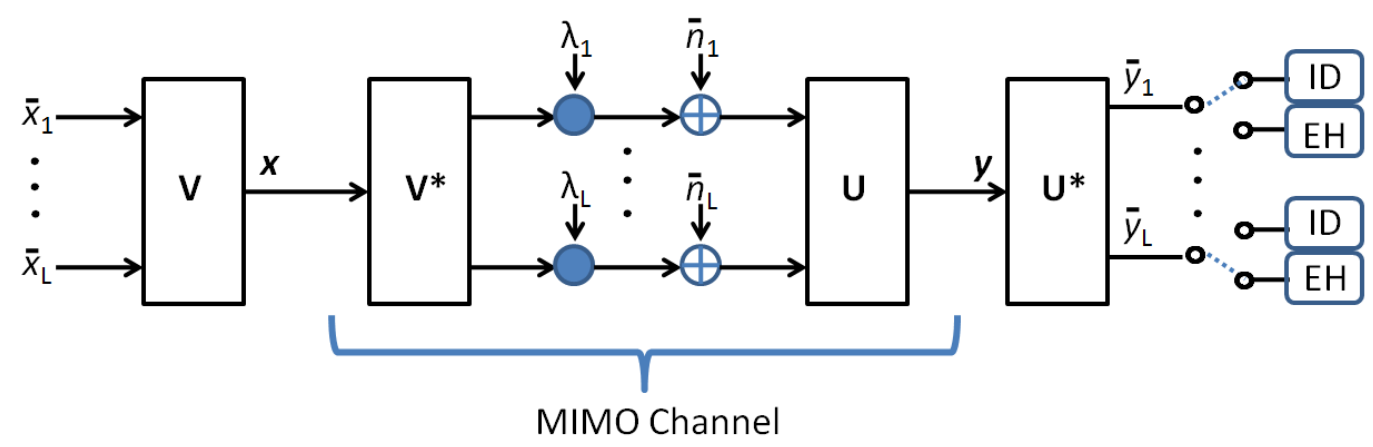

Fig. 2. Schematic example of the SVD of the MIMO channel into $L$ parallel AWGN channels.

the selected active receive antenna set and the corresponding channel from the source to the destination are respectively denoted with $\chi \in\left\{1, \cdots, N_{R}\right\}$ and $\mathbf{H}_{\chi} \in C^{N \times N_{T}}$, where $N=|\chi|$.

Let $\mathbf{x} \in C^{N_{T} \times 1}$ and $\mathbf{n} \in C^{N \times 1}$ denote the transmit signal vector and circularly symmetric complex additive white Gaussian noise (AWGN) vector with zero mean and unit variance, respectively. The received signal is given by

$$
\mathbf{y}=\mathbf{H}_{\chi} \mathbf{x}+\mathbf{n},
$$

where $E\left[\mathbf{x x}^{H}\right]=\mathbf{Q}_{\chi}$, with $\mathbf{Q}_{\chi}$ being the transmit covariance matrix. Therefore, with the selected receive antenna set $\chi$ $\left(\mathbf{H}_{\chi} \in C^{N \times N_{T}}\right)$, the mutual information in the MIMO SWIPT system with SS-based receiver is formulated as [6]

$$
I(\mathbf{x} ; \mathbf{y})=\log \operatorname{det}\left(\mathbf{I}_{N}+\mathbf{H}_{\chi} \mathbf{Q}_{\chi} \mathbf{H}_{\chi}^{H}\right) .
$$

Fig. 2 provides an illustrative example of the MIMO channel decomposition for potentially conveying information and energy. With a fixed active receive antenna set $\chi$, the SVD-based transformation of the channel matrix $\mathbf{H}_{\chi}$ can be expressed as $\mathbf{H}_{\chi}=\mathbf{U} \boldsymbol{\Sigma} \mathbf{V}^{H}$, where $\mathbf{U} \in C^{N \times N}$ and $\mathbf{V} \in C^{N_{T} \times N_{T}}$ correspond to unitary matrices whilst $\boldsymbol{\Sigma} \in C^{N \times N_{T}}$ is a diagonal matrix containing the singular values of the channel matrix $\mathbf{H}_{\chi}, \lambda_{i}(\chi)$, respectively. Hence, the MIMO channel (with the selected receive antenna set $\chi$ ) is decomposed into $L$ parallel SISO channels with

$$
\tilde{y}_{i}=\lambda_{i}(\chi) \tilde{x}_{i}+\tilde{n}_{i},
$$

where $\tilde{n}_{i}$ is AWGN for the $i$-th parallel SISO channel. Considering that SVD is a unitary transformation of the MIMO channel, $\tilde{n}_{i}$ follows the same distribution with that of $n_{i}$. Therefore, as illustrated in Fig. 2, the output of each eigenchannel is connected either to the information decoding (ID) circuit or to the $\mathrm{EH}$ rectification circuit.
In order to depict the above notion, we use the binary variable $\alpha_{i}$ in order to indicate whether the $i$-th eigen-channel is used for data transmission $\left(\alpha_{i}=1\right)$ or energy transfer $\left(\alpha_{i}=0\right)$. Therefore, the sum-rate of the $L$ parallel SISO channels with the selected receive antenna set $\chi$ is given by

$$
C=\sum_{i=1}^{L} \log _{2}\left(1+\alpha_{i} p_{i} \lambda_{i}(\chi)\right)
$$

where $p_{i}$ is the power allocated to the $i$-th eigen-channel for data transmission. On the other hand, the total harvested energy at the receiver can be written as

$$
E=\sum_{i=1}^{L} \eta\left(1-\alpha_{i}\right) p_{i} \lambda_{i}(\chi)
$$

where $\eta$ represents the loss from the energy transducer conversion of the harvested energy to electrical energy.

For the SS-based MIMO SWIPT system under consideration, the power model should account for the the power consumption of the entire transmit-receiver chain. This includes the impact of the transmit power, circuit power, as well as RF energy harvester. It can be argued that the latter consumes small amounts of power, and thus may not significantly affect the EE of the system. On the other hand, it is intuitive to infer that the system power consumption is in part may be compensated by the transferred energy. As a result, similar to the approach in [7], here, the harvested energy is taken into consideration. In particular, the total power consumption is formulated using a linear power model as follows

$$
P=\zeta P_{T}+P_{C}-E
$$

where $\zeta, P_{T}$ and $P_{C}$ are respectively represent the reciprocal of drain efficiency of the power amplifier, transmit power, and the total circuit power consumption (note that $E$ is defined in 
(5)). The minus sign in (6) implies that the receiver is able to harvest a portion of the radiated power from the transmitter. The total transmission power $P_{T}$ correspond to the sum of all powers allocated to the eigen-channels, i.e. $P_{T}=\sum_{i=1}^{L} p_{i}$. Further, the total circuit power consumption $P_{C}$ can be split into static and dynamic parts based on the configurations of the active links. In this work, the transmit-dependent circuit power consumption is modeled as a linear function of the number of active antennas using

$$
P_{C}=P_{s t a}+P_{a n t}^{B S} N_{T}+P_{a n t} N=\bar{P}_{s t a}+P_{a n t} N
$$

where $\bar{P}_{\text {sta }}=P_{\text {sta }}+P_{a n t}^{B S} N_{T}$ is the static circuit power at the transmitter and $P_{a n t} N$ denotes the dynamic power consumption which is proportional to the number of active receive antennas in a SS-based MIMO SWIPT system.

The EE can be defined as the total number of delivered bits per unit energy. Hence, we express the EE of the SS-based MIMO SWIPT system with receive antenna set $\chi$ using

$\psi_{E E} \triangleq \frac{C}{P}=\frac{\sum_{i=1}^{L} \log _{2}\left(1+\alpha_{i} p_{i} \lambda_{i}(\chi)\right)}{\zeta P_{T}+\bar{P}_{s t a}+P_{a n t} N-\sum_{i=1}^{L} \eta\left(1-\alpha_{i}\right) p_{i} \lambda_{i}(\chi)}$,

where $C$ is the corresponding sum-rate of the $L$ parallel SISO channels with the selected receive antenna set $\chi$.

The objective of this paper is to maximize the EE of a SSbased MIMO SWIPT system whilst meeting two important QoS constraints in terms of minimum sum-rate and harvested energy. The corresponding optimization problem can be mathematically formulated as

$$
\begin{aligned}
& \max _{\alpha_{i}, p_{i}, \chi} \frac{\sum_{i=1}^{L} \log _{2}\left(1+\alpha_{i} p_{i} \lambda_{i}(\chi)\right)}{\zeta P_{T}+} \bar{P}_{\text {sta }}+P_{\text {ant }} N-\sum_{i=1}^{L} \eta\left(1-\alpha_{i}\right) p_{i} \lambda_{i}(\chi) \\
& \text { s.t. } \quad \sum_{i=1}^{L} \log _{2}\left(1+\alpha_{i} p_{i} \lambda_{i}(\chi)\right) \geq R_{\text {min }}, \\
&\left.\sum_{i=1}^{L} \eta\left(1-\alpha_{i}\right) p_{i} \lambda_{i}(\chi)\right) \geq E_{\text {min }}, \\
& \sum_{i=1}^{L} p_{i} \leq P_{\max }, \\
& p_{i} \geq 0, \alpha_{i} \in\{0,1\}, \forall i \in \mathcal{L},
\end{aligned}
$$

where $R_{\min }, E_{\min }$ and $P_{\max }$ are respectively the minimum sum-rate, minimum harvested energy, and maximum transmit power constraints. The minimum energy requirement is defined as the minimum additional amount of harvested energy in one transmission cycle in cases where energy storage is not viable. Otherwise, $E_{\min }$ corresponds to the required amount of energy of the energy harvester to function in the next transmission cycle. Moreover, (12) and (13) are the constraints for the allocated power and SS indicators.

It is easy to see that the EE optimization problem involves binary and continuous variables as well as non-linear functions; hence it belongs to the class of mixed-integer nonlinear optimization problems. Furthermore, jointly selecting the "best" receive antenna set $\chi$, eigen-channel assignment $\alpha_{i}$, and power allocation $p_{i}$ makes the problem (9)-(13) nonconvex and hence intractable to tackle directly. Consequently, in the following sections, we develop joint antenna selection and SS approaches in order to maximize EE. Since $\chi$ affects the EE optimization problem in a comprehensive manner, i.e., $\chi$ relates to the channel matrix (effective channel gain), the dynamic spatial assignment and power allocation, solving $\chi$ jointly with $\alpha_{i}$ and $p_{i}$ is not straightforward. Nevertheless, for any optimization problems, it is possible to tackle the problem over some of the variables and then over the remaining ones [8]. Therefore, we will optimize the eigen-channel assignment $\alpha_{i}$ and power allocation $p_{i}$ at first (inner-layer process) under fixed receive antenna set $\chi$. Thereafter, we propose a strategy to determine the optimal receive antenna set $\chi$ in order to further improve the achievable EE (outer-layer process).

\section{Joint Eigen-Channel Assignment And Power ALLOCATION}

For ease of description, we omit $\chi$ in the subscript in this section. Even though the antenna set is fixed here, the problem still belongs to the class of mixed-integer non-linear optimization problems, which is very difficult to solve directly. Similar to the approximation widely used in the context of OFDMA resource allocation [9], our eigen-channel assignment and power allocation problem can be approximated as

$$
\begin{gathered}
\max _{\tilde{\alpha}_{i}, p_{i}} \frac{\sum_{i=1}^{L} \tilde{\alpha}_{i} \log _{2}\left(1+\frac{p_{i} \lambda_{i}}{\tilde{\alpha}_{i}}\right)}{\zeta \sum_{i=1}^{L} p_{i}+\bar{P}_{\text {sta }}+P_{a n t} N-\sum_{i=1}^{L} \eta\left(1-\tilde{\alpha}_{i}\right) p_{i} \lambda_{i}} \\
\text { s.t. } \quad \sum_{i=1}^{L} \tilde{\alpha}_{i} \log _{2}\left(1+\frac{p_{i} \tilde{\lambda}_{i}}{\tilde{\alpha}_{i}}\right) \geq R_{\text {min }}, \\
\sum_{i=1}^{L} \eta\left(1-\tilde{\alpha}_{i}\right) p_{i} \lambda_{i} \geq E_{\text {min }}, \\
\sum_{i=1}^{L} p_{i} \leq P_{\max }, \\
p_{i} \geq 0, \tilde{\alpha}_{i} \in[0,1], \forall i \in \mathcal{L} .
\end{gathered}
$$

It should be noted that when $\tilde{\alpha}_{i}$ approaches zero, $\tilde{\alpha}_{i} \log _{2}(1+$ $\frac{p_{i} \tilde{\lambda}_{i}}{\tilde{\alpha}_{i}}$ ) also tends to zero, which is similar to setting $\alpha_{i}$ to zero , i.e., the $i$-th eigen-channel is nearly not assigned for data transmission but for $\mathrm{EH}$. On the other hand, when $\tilde{\alpha}_{i}$ is close to one, $\tilde{\alpha}_{i} \log _{2}\left(1+\frac{p_{i} \tilde{\lambda}_{i}}{\tilde{\alpha}_{i}}\right)$ is close to $\log _{2}\left(1+\alpha_{i} p_{i} \lambda_{i}\right)$, which indicates that the $i$-th eigen-channel is almost entirely assigned for data transmission. Therefore, when $\tilde{\alpha}_{i}$ is close to zero or one, the approximation becomes precise. As a result, we will use $\tilde{\alpha}_{i}$ instead of $\alpha_{i}$ to represent the eigen-channel assignment for either data transmission or $\mathrm{EH}$ of the $i$-th channel in a modified EE optimization problem. On the other hand, the solution of problem (14)-(18) may provide fractional eigenchannel assignment $\tilde{\alpha}_{i}^{*}$, and hence the proposed transformation provides an upper-bound solution.

It should also be noted that the optimal solution involves eigen-channel assignment $\tilde{\alpha}_{i}^{*}$ are not strictly either 0 or 1 . To get a feasible solution to the original optimization problem, we need to round the possibly fractional eigen-channel assignment $\tilde{\alpha}_{i}^{*}$ to 0 or 1 and then perform the power allocation algorithm to get the maximum "reasonable" EE for the round-off $\tilde{\alpha}_{i}^{\text {round }}$. On the other hand, it has been shown in [10] that the optimal 
$\tilde{\alpha}_{i}^{*}$ mostly tends to either 0 or 1 , hence this enables us to precisely solve the original problem.

Since the optimization problem in (14)-(18) involves a nonlinear fractional programming problem, it is non-convex and difficult to solve directly. However, given that the Dinkelbach method is an efficient method to tackle such problems [11], we therefore can apply it to solve our non-convex nonlinear fractional programming problem. Specifically, we transform the fractional form objective function into a numeratordenominator subtractive form using the following proposition.

Proposition 1: The maximum achievable EE $\beta^{*}=\psi_{E E}^{*}$ can be obtained provided that

$$
\begin{gathered}
\max _{\mathbf{p}, \tilde{\boldsymbol{\alpha}}} U_{R}(\mathbf{p}, \tilde{\boldsymbol{\alpha}})-\beta^{*} U_{T}(\mathbf{p}, \tilde{\boldsymbol{\alpha}}) \\
=U_{R}\left(\mathbf{p}^{*}, \tilde{\boldsymbol{\alpha}}^{*}\right)-\beta^{*} U_{T}\left(\mathbf{p}^{*}, \tilde{\boldsymbol{\alpha}}^{*}\right)=0
\end{gathered}
$$

for $U_{R}(\mathbf{p}, \tilde{\boldsymbol{\alpha}}) \geq 0$ and $U_{T}(\mathbf{p}, \tilde{\boldsymbol{\alpha}}) \geq 0$, where

$$
\begin{aligned}
& U_{R}(\mathbf{p}, \tilde{\boldsymbol{\alpha}})=\sum_{i=1}^{L} \log _{2}\left(1+\alpha_{i} p_{i} \lambda_{i}\right), \\
& U_{T}(\mathbf{p}, \tilde{\boldsymbol{\alpha}})=\zeta \sum_{i=1}^{L} p_{i}+P_{\text {sta }}+P_{\text {ant }} N \\
& -\sum_{i=1}^{L} \eta\left(1-\alpha_{i}\right) p_{i} \lambda_{i} \\
& \beta^{*}=\frac{U_{R}\left(\mathbf{p}^{*}, \tilde{\boldsymbol{\alpha}}^{*}\right)}{U_{T}\left(\mathbf{p}^{*}, \tilde{\boldsymbol{\alpha}}^{*}\right)}, \\
& \text { and } \mathbf{p}=\left[p_{0}, p_{1}, \cdots, p_{L}\right], \tilde{\boldsymbol{\alpha}}=\left[\tilde{\alpha}_{1}, \tilde{\alpha}_{2}, \cdots, \tilde{\alpha}_{L}\right] \text {. }
\end{aligned}
$$

Proposition 1 provides an adequate and compulsory condition for developing the optimal resource allocation scheme. In particular, based on the original optimization problem with a fractional form-objective function, an equivalent optimization problem with a subtractive form-objective function (e.g. $\left.U_{R}(\mathbf{p}, \tilde{\boldsymbol{\alpha}})-\beta^{*} U_{T}(\mathbf{p}, \tilde{\boldsymbol{\alpha}})\right)$ can be found such that the same solution can be achieved for both optimization problems. Moreover, [11] further implies that the optimal solution is achieved with equality in (19), and thus we could use this equality condition to validate the optimality of the solution. Hence, rather than tackling the original fractional formobjective function (14)-(18), we develop a resource allocation algorithm for the equivalent subtractive form-objective function whilst meeting the conditions in Proposition 1. The proposed algorithm is summarized in Table I.

As shown in Table I, the pivotal stage for the proposed Dinkelbach method-based solution is to develop an intermediate resource allocation policy in order to solve the following fixed $\beta$ optimization problem (step 3 in Table I),

$$
\begin{aligned}
& \max _{\tilde{\alpha}_{i}, p_{i}} \sum_{i=1}^{L} \tilde{\alpha}_{i} \log _{2}\left(1+\frac{p_{i} \lambda_{i}}{\tilde{\alpha}_{i}}\right) \\
& -\beta\left(\zeta \sum_{i=1}^{L} p_{i}+\bar{P}_{\text {sta }}+P_{\text {ant }} N-\sum_{i=1}^{L} \eta\left(1-\tilde{\alpha}_{i}\right) p_{i} \lambda_{i}\right) \\
& \text { s.t. } \quad \sum_{i=1}^{L} \tilde{\alpha}_{i} \log _{2}\left(1+\frac{p_{i} \lambda_{i}}{\tilde{\alpha}_{i}}\right) \geq R_{\text {min }},
\end{aligned}
$$

1) Initialize $\beta=0$, and $\delta$ as the stopping criterion;

\section{2) REPEAT}

3) For a given $\beta$, solve (24)-(28) to obtain the eigen -channel assignment and power allocation $\{\mathbf{p}, \tilde{\boldsymbol{\alpha}}\}$;

4) IF $U_{R}(\mathbf{p}, \tilde{\boldsymbol{\alpha}})-\beta U_{T}(\mathbf{p}, \tilde{\boldsymbol{\alpha}}) \leq \delta$

5) Convergence $=$ TRUE;

6) $\quad \operatorname{RETURN}\left\{\mathbf{p}^{*}, \tilde{\boldsymbol{\alpha}}^{*}\right\}=\{\mathbf{p}, \tilde{\boldsymbol{\alpha}}\}, \beta^{*}=\frac{U_{R}(\mathbf{p}, \tilde{\boldsymbol{\alpha}})}{U_{T}(\mathbf{p}, \tilde{\boldsymbol{\alpha}})}$ 7) ELSE

8) $\quad$ Set $\beta=\frac{U_{R}(\mathbf{p}, \tilde{\boldsymbol{\alpha}})}{U_{T}(\mathbf{p}, \tilde{\boldsymbol{\alpha}})}$ and $n=n+1$,

\section{9) END IF} Convergence $=$ FALSE;

10) UNTIL Convergence $=$ TRUE.

TABLE I

PROPOSED ITERATIVE RESOURCE ALLOCATION ALGORITHM BASED ON DINKELBACH METHOD

$$
\begin{aligned}
& \left.\sum_{i=1}^{L} \eta\left(1-\tilde{\alpha}_{i}\right) p_{i} \lambda_{i}\right) \geq E_{\text {min }}, \\
& \sum_{i=1}^{L} p_{i} \leq P_{\max }, \\
& p_{i} \geq 0, \tilde{\alpha}_{i} \in[0,1], \forall i \in \mathcal{L} .
\end{aligned}
$$

Proposition 2: For a given parameter $\beta$, the objective function (24) is strictly and jointly concave in $\tilde{\alpha}_{i}$ and $p_{i}$.

Therefore, since the objective function is a concave function and the constraint set is also convex, the modified optimization problem in (24)-(28) is in the standard form of a convex programming problem that can be solved by standard numerical methods such as the interior-point method [12]. Hence, problem (14)-(18) can be successfully solved by the proposed convex programming based Dinkelbach method.

\section{Active Receive Antenna Selection}

For the SS-based MIMO SWIPT system, it is intuitive to conclude that the optimal receive antenna selection strategy is the exhaustive search. Specifically, for each possible receive antenna set $\chi \in\left\{1,2, \cdots, N_{R}\right\}$, we obtain the EE based on the proposed joint eigen-channel assignment and power allocation algorithm in section III, and then select the optimal active receive antenna set as

$$
\chi^{\text {opt }}=\arg \underset{\chi \in\left\{1,2, \cdots, N_{R}\right\}}{\max } \psi_{E E}(\chi) .
$$

However, the computational complexity of this exhaustive search scheme is too high to implement in practice. Therefore, developing low-complexity approaches with low-complexity is necessary per discussed in the following.

Since the optimal solution has to calculate the EE for all possible antenna sets, this implies that the antenna selection process is separated from the eigen-channel assignment and power allocation procedures. In other words, the receive antenna selection process is not connected with SS (eigenchannel) for ID and EH. Therefore, with a given number of receive antenna $N=|\chi|$, our aim is to select the set of receive antennas that maximizes the $\mathrm{EE}$ of the system which is used for either ID or EH. We therefore arrive at the following.

Proposition 3. With a fixed transmit power $P$, the maximum 
EE of the system where ID and EH are operated at the same time, can be achieved using the following multi-objective optimization problem

$$
\begin{gathered}
\max _{|\chi|=N, \boldsymbol{Q}_{\chi}>0}\left\{\frac{\log \operatorname{det}\left(\boldsymbol{I}_{N}+\boldsymbol{H}_{\chi} \boldsymbol{Q}_{\chi} \boldsymbol{H}_{\chi}^{H}\right)}{\tilde{P}}, \frac{\theta \eta \operatorname{tr}\left(\boldsymbol{H}_{\chi} \boldsymbol{Q}_{\chi} \boldsymbol{H}_{\chi}^{H}\right)}{\tilde{P}}\right\} \\
\text { s.t. } \quad \operatorname{tr}\left(\boldsymbol{Q}_{\chi}\right)=P .
\end{gathered}
$$

where $\tilde{P}=\zeta \operatorname{tr}\left(\boldsymbol{Q}_{\chi}\right)+\bar{P}_{\text {sta }}+P_{\text {ant }} N$ is the total power, $\frac{\log \operatorname{det}\left(\boldsymbol{I}_{N}+\boldsymbol{H}_{\chi} \boldsymbol{Q}_{\chi} \boldsymbol{H}_{\chi}^{H}\right)}{\zeta \operatorname{tr}\left(\boldsymbol{Q}_{\chi}\right)+\bar{P}_{\text {sta }}+P_{a n t} N}$ and $\frac{\theta \eta \operatorname{tr}\left(\boldsymbol{H}_{\chi} \boldsymbol{Q}_{\chi} \boldsymbol{H}_{\chi}^{H}\right)}{\zeta \operatorname{tr}\left(\boldsymbol{Q}_{\chi}\right)+\bar{P}_{\text {sta }}+P_{a n t} N}$ are respectively representing the EE of the conventional ID MIMO system and the EH MIMO system.

Considering equal transmit power allocation at each antenna, we can transform (30) using

$$
\begin{aligned}
\max _{\chi:|\chi|=N, P>0} & \left\{\frac{\log \operatorname{det}\left(\mathbf{I}_{N}+\frac{P}{N} \mathbf{H}_{\chi} \mathbf{H}_{\chi}^{H}\right)}{\tilde{P}}, \frac{\theta \eta \frac{P}{N} \operatorname{tr}\left(\mathbf{H}_{\chi} \mathbf{H}_{\chi}^{H}\right)}{\tilde{P}}\right\} \\
= & \max _{\chi:|\chi|=N}\left\{\operatorname{det}\left(\mathbf{H}_{\chi} \mathbf{H}_{\chi}^{H}\right), \operatorname{tr}\left(\mathbf{H}_{\chi} \mathbf{H}_{\chi}^{H}\right)\right\} .
\end{aligned}
$$

However, calculating the channel matrix determinant or the trace of the channel matrix requires a large number of computations, especially when the system is equipped with a large number of antennas. Therefore, instead of directly applying determinant or trace operations to the channel matrix, we here incorporate the Frobenius-norm of the channel matrix in order to reduce the computational complexity. Although the Frobenius-norm of the channel cannot directly characterize the capacity and harvested energy precisely, it is related to the throughput and harvested energy by demonstrating the overall energy of the channel [13]. As a result, the selection criterion for the SS-based MIMO SWIPT system is based on

$$
\operatorname{sort}_{1 \leq n \leq N}\left\|\mathbf{h}_{n}\right\|_{F}^{2}
$$

where $\mathbf{h}_{n}$ denotes the $n$-th row of the channel matrix $\mathbf{H}$, which represents the channel quality of the $n$-th receive antenna. After sorting, the receive antenna set is selected from the first $N_{R}$ rows of the sorted matrix. We then only need to perform the proposed eigen-channel assignment and power allocation algorithm to maximize EE. This process is repeated until all the receive antenna number has been investigated.

\section{Simulation Results}

In our simulations, the total number of transmit and receive antennas are respectively $N_{T}=8$ and $N_{R}=8$. In addition, the drain efficiency of the power amplifier $\zeta$ is set to $38 \%$ whereas the EH efficiency is taken to be $\eta=10 \%$. The static circuit power at the transmitter $P_{s t a}$ is assumed to be $5 \mathrm{~W}$ and the dynamic power consumption proportional to the number of antennas $P_{a n t}$ is set to be $1 \mathrm{~W}$.

In the first simulation, the convergence behavior of the proposed convex programming-based Dinkelbach method (DMCVX) is studied. For convenience, we denote the full-searchbased approach as FSA. As shown in Fig. 3, DM-CVX converges to a stable value which is very close to the FSA. This demonstrates that the proposed algorithm can efficiently approach the optimal EE. Moreover, it is observed that there is a drop on EE at the 20-th iteration for DM-CVX. This is

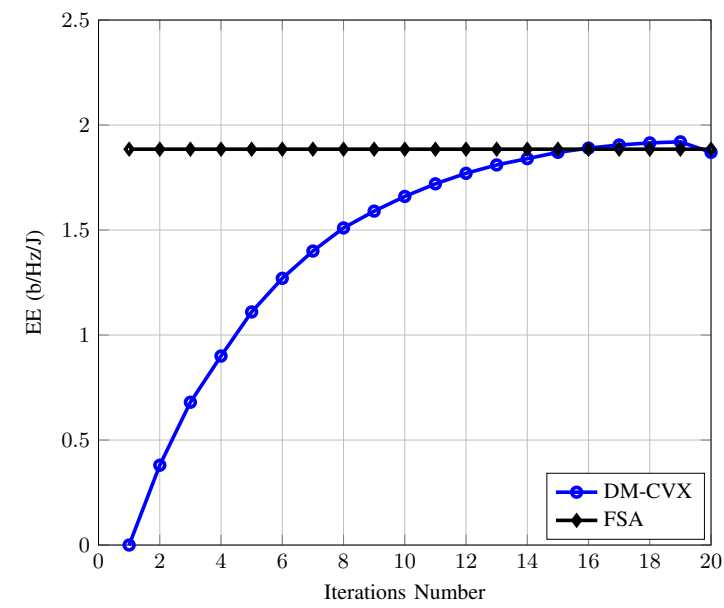

Fig. 3. Convergence behavior of the proposed eigen-channel assignment and power allocation approaches (fixed antenna set).

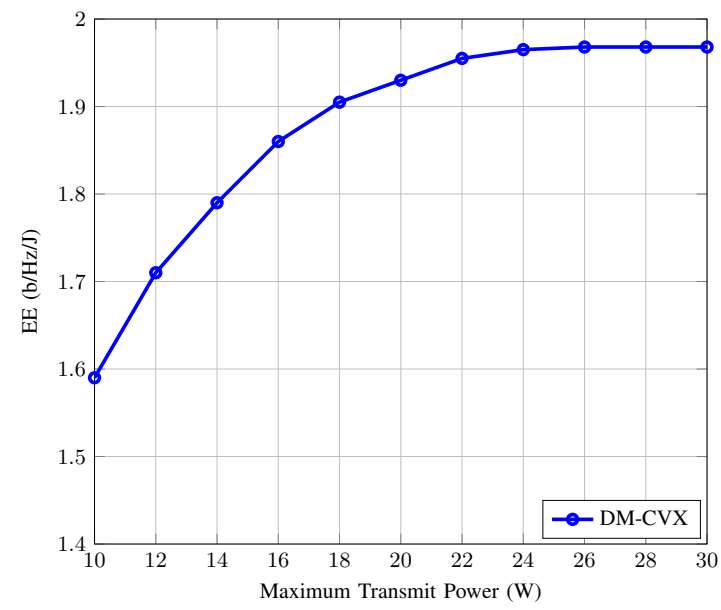

Fig. 4. The performance of the proposed Dinkelbach method-based joint eigen-channel assignment and power allocation algorithm with different static circuit power (EE vs transmit power constraint).

because after this solution converges, the possibly fractional $\tilde{\alpha}_{i}^{*}$ is rounded to either 0 or 1 and the proposed power allocation algorithm has been performed again to get the maximum $\mathrm{EE}$ for the round-off case. This result further coincides with our theoretical findings where the proposed scheme is upperbound solutions due to the relaxation of $\alpha_{i}$.

In the next simulation, DM-CVX under different constraints is evaluated and presented in Fig. 4 and Fig. 5. As can be seen from Fig. 4, the EE achieved by DM-CVX is monotonically non-decreasing with respect to the maximum transmit power constraint $P_{\max }$. Particularly, the EE increases in the lower transmit power constraint region, i.e., $10<P_{\max }<20$ $\mathrm{W}$, and then saturates when $P_{\max }>20 \mathrm{~W}$ due to the fact that a balance between the system EE and the total power consumption can be achieved. We also investigate the $\mathrm{EE}$ versus the minimum required harvested energy. As shown in Fig. 5, the optimal EE is the same up to a certain minimum required harvested energy, but drops afterwards.

Finally, we evaluate the performance of the proposed normbased receive antenna selection algorithm for the SS-based MIMO SWIPT system. To show the EE gain, we compare 


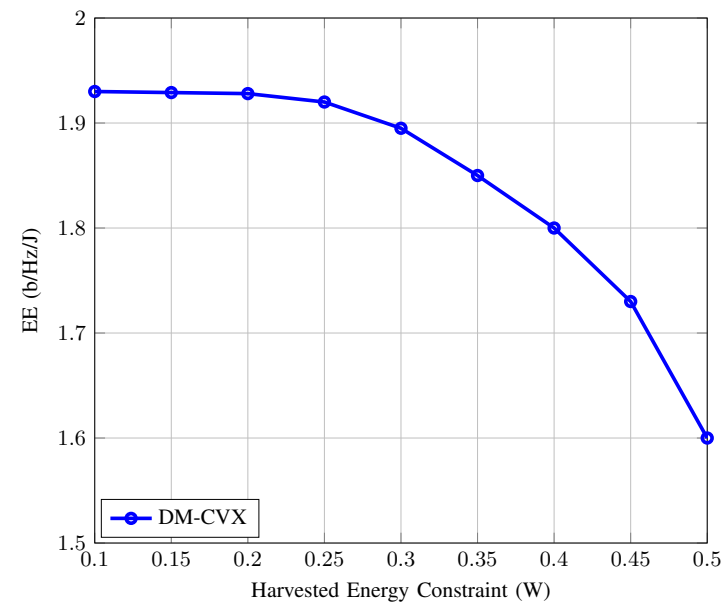

Fig. 5. The performance of the proposed Dinkelbach method-based joint eigen-channel assignment and power allocation algorithm with different static circuit power (EE vs EH constraint).

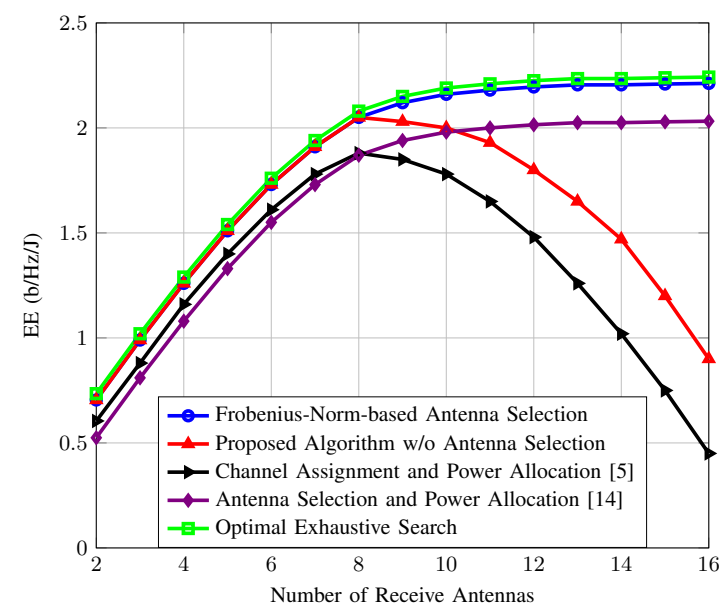

Fig. 6. Energy efficiency versus the maximum transmit power allowance for the proposed extended BC-MAC duality-based EE maximization algorithm with different minimum required power transfer.

with the scheme that maximizes the $\mathrm{EE}$ but without $\mathrm{EH}$ [14], and the scheme in [5] which minimizes the transmit power in SS-based MIMO SWIPT system without considering antenna selection strategy. We also show the performance of our proposed scheme but without antenna selection. In other words, this scheme always employs all the receive antennas. As shown in Fig. 6, the EE achieved by our proposed norm-based antenna selection approach is monotonically nondecreasing with respect to the number of active receive antenna $N$. Particularly, the EE increases linearly with an increasing $N$ in the lower region, i.e., $2<N<8$, and then saturates when $N>10$ as a balance between the system EE and the spatial (eigen-channel) gain is achieved. Furthermore, the EE achieved by the proposed norm-based selection approach outperform the EE achieved in [14] and [5], and is very close to the optimal exhaustive search approach; but with a lower complexity. Interestingly, for the case of higher total number of active receive antennas, i.e., $N \geq 10$, the EE achieved by the proposed algorithm without considering antenna selection is lower than that of the scheme proposed in [14]. This implies that the EE gain achieved by EH cannot compensate the cost of activating redundant antennas.

\section{CONCLUSIONS}

In this paper, we address the EE optimization problem for MIMO SWIPT system with SS-based receiver. Considering a practical linear power model where the number of active receive antennas, transmit power, and power transfer are taken into consideration, our target is to maximize the EE whilst satisfying certain constraints in terms of minimum sum-rate and power transfer. The EE optimization problem, which involves joint optimization of the eigen-channel assignment, power allocation, and active receive antenna set, is non-convex, and thus extremely difficult to tackle directly. Hence, to obtain a feasible solution for this problem, we propose to separate the antenna selection procedure with the eigen-channel assignment and power allocation operation. In particular, under fixed receive antenna set, near-optimal convex programming-based Dinkelbach method is developed. We then study antenna selection to further explore the achievable EE and accordingly provide optimal exhaustive search and Frobenius-norm-based dynamic selection schemes. Numerical results illustrate that the proposed joint antenna selection and SS-based approach outperforms state-of-the-art schemes in terms of improving the EE performance of the MIMO SWIPT system.

\section{REFERENCES}

[1] E. Hossain, M. Rasti, H. Tabassum, and A. Abdelnasser, "Evolution toward 5G multi-tier cellular wireless networks: An interference management perspective," IEEE Trans. Wireless Commun., vol. 21, no. 3, pp. 118-127, June 2014.

[2] L. R. Varshney, "Transporting information and energy simultaneously," in Proc. 2008 IEEE Int. Symp. Inf. Theory, July 2008, pp. 1612-1616.

[3] R. Zhang and C. K. Ho, "MIMO broadcasting for simultaneous wireless information and power transfer," IEEE Trans. Wireless Commun., vol. 12, no. 5, pp. 1989-2001, May 2013.

[4] A. A. Nasir, X. Zhou, S. Durrani, and R. A. Kennedy, "Relaying protocols for wireless energy harvesting and information processing," IEEE Trans. Wireless Commun., vol. 12, no. 7, pp. 3622-3636, July 2013.

[5] S. Timotheou, I. Krikidis, S. Karachontzitisand, and K. Berberidis, "Spatial domain simultaneous information and power transfer for MIMO channels," IEEE Trans. Wireless Commun., vol. 14, no. 8, pp. 41154128, Aug. 2015.

[6] A. Goldsmith, Wireless Communications. Stanford University, 2005.

[7] D. W. K. Ng, E. S. Lo, and R. Schober, "Robust beamforming for secure communication in systems with wireless information and power transfer," IEEE Trans. Wireless Commun., vol. 13, no. 8, pp. 4599-4615, Aug. 2014.

[8] S. Boyd and L. Vandenberghe, Convex Optimization. Cambridge University Press, Cambridge, UK, 2004.

[9] C. Xiong, G. Li, S. Zhang, Y. Chen, and S. Xu, "Energy- and spectralefficiency tradeoff in downlink OFDMA networks," IEEE Trans. Wireless Commun., vol. 10, no. 11, pp. 3874-3886, Nov. 2011.

[10] W. Yu and J. M. Coffi, "FMDA capacity of Gaussian multiple-access channel with ISI," IEEE Trans on Commun., vol. 50, no. 1, pp. 102-111, Jan. 2002.

[11] W. Dinkelbach, "On nonlinear fractional programming," Management Science, vol. 13, pp. 492-498, Mar. 1967.

[12] S. Boyd, Branch and Bound Methods. Stanford University.

[13] Z. Shen, R. Chen, J. G. Andrews, R. W. Heath, and B. L. Evans, "Low complexity user selection algorithms for multiuser MIMO systems with block diagonalization," IEEE Trans. Sig. Lett., vol. 54, no. 9, pp. 36583663, Sep. 2006.

[14] J. Tang, D. K. C. So, E. Alsusa, and K. A. H. A. Shojaeifard, "On the energy efficiency-spectral efficiency trade-off in MIMO-OFDMA broadcast channels," IEEE Trans. Veh. Tech., vol. 65, no. 7, pp. 51855199, July 2016. 